\title{
Cloud Computing for hand-held Devices:Enhancing Smart phones viability with Computation Offload
}

\author{
Mohd. Abdul Salam \\ (Research Scholar, CMJ University, Shillong, Meghalaya)
}

\begin{abstract}
Cloud computing is Modern day's wonder. It is not a product but a service, which provides shared resources, software, and information to computers and other devices like smart phones as a utility over a network mainly internet [1]. Resources namely memory, storage space, processor, etc are not available at user's end explicitly. Service providers own these resources and user access them via the Internet. It comes with many advantages for business like lower operation cost, low capital investment, shorter startup time for new services, lower maintenance cost. Cloud computing is a boon for shifting computing from desktops to cloud. Now the new paradigm should be cloud computing for mobile users. The limitations for mobile cloud computing are limited availability of energy and wireless bandwidth. Mobile Cloud Computing combines cloud computing and mobile resources to overcome obstacles related to the performance (like battery life and bandwidth), environment (heterogeneity, scalability, and availability), and security (reliability, security and privacy). In this paper it is discussed how cloud computing may provide energy saving to mobile users and hence increasing the battery life of the mobile.
\end{abstract}

Keywords-Computation Offloading, Mobile Cloud Computing, Cloud Computing.

\section{Introduction}

Nowadays many users use smart phones as a computing platform instead of a computer. Users need "Longer battery life" as an important constraint while operating a smart phone.

There are a number of computation intensive applications which have to be performed on the cloud rather than on a mobile system to save energy and increase battery lifetime of the mobile.

\section{Computation Offload To Save Energy And Increase Battery Life}

It is similar to renowned client-server architecture. Cloud computing is different from the client-server computing model in aspects of adoption of virtualization in cloud computing. Through virtualization the cloud vendors can execute random programs given by the users. Mobile users can use the computation cycles provided by the cloud vendors, so that amount of computation on mobile systems reduce to a great extent and resultantly increase battery life. So cloud computing provides energy saving as a service to mobile users particularly called as "Computation Offloading".

Virtualization allows applications from different mobile users to run on different virtual machines. Hence it provides necessary advantages like security and protection from intrusion.

The energy saved by computation offloading depends on the wireless bandwidth, the amount of data to be transmitted and the amount of computation to be performed. There is no need to send all the data to the service provider. The cloud stores data and performs computation on stored data. (e.g. Amazon's S3 (Simple Storage Service) can store data and Amazon's Elastic Compute Cloud

(EC2) can be used to perform computation on the data stored using S3). This reduces the amount of data to be transmitted over the wireless network drastically. Very small data to be transmitted and very large computation speed imply that the energy can be saved.

\section{Advantages:}

(i) Virtualization enables the cloud vendors to execute different programs given by the user.

(ii) Virtualization allows different applications to be mutually exclusive as virtualization allows applications from different mobile users to run on different virtual machines.

\section{Disadvantages:}

(i) Energy saved directly depends on the wireless bandwidth of the network over which the data has to be sent. If the bandwidth is more, energy saved will be more because the data can be transferred to cloud quickly.

(ii) Security and Privacy: MCC is vulnerable to various attacks like Phishing by which one can illegally gain another's passwords and other sensitive information. 


\section{Solutions to the challenges:}

(i) Encrypting data before storing them in the cloud.

(ii) Performing some steganographic techniques to transform data so that operations can be performed over data without exposing them.

\section{Downsides of the solutions proposed:}

Performing encryption or steganographic techniques before sending data to the cloud requires some additional processing on the mobile system and consumes additional energy.

Hoang T. Dinh, Chonho Lee, Dusit Niyato, and Ping Wang stated that Mobile Cloud Computing (MCC) is an integration of mobile web technology and cloud computing [2][3].Mobile users can access various applications and services through MCC. Storage and data processing services in cloud are provided by MCC. There is no need for the mobile devices to possess powerful configuration like processor speed, RAM, etc as all the processes run on cloud itself.

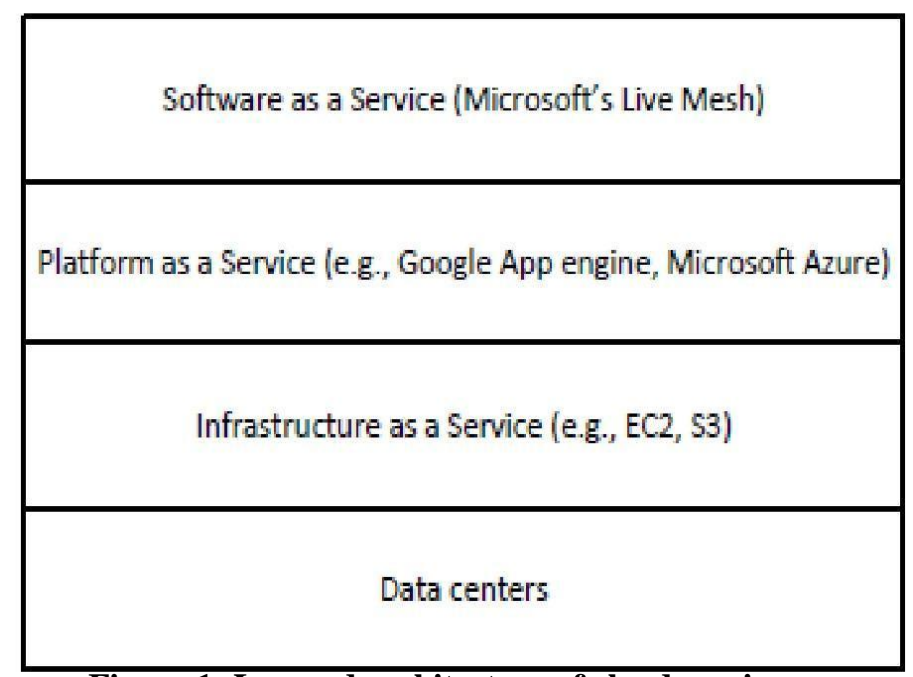

Figure 1: Layered architecture of cloud services

Figure 1 depict that cloud services have a layered architecture. Upper layers are composed of Infrastructure as a Service (IaaS), Platform as a Service (PaaS), and Software as a Service (SaaS).

Data Centers Layer: Gives access to client for the required hardware and infrastructure for cloud.

Infrastructure as a Service (IaaS): It is built over the data center layer. It avails different services like storage space, hardware, network units and servers. Client pays for the services on a basis of Pay per use.

Software as a Service (SaaS): The users can use various applications and services provided by the cloud vendors remotely through the Internet and pay on a Pay per - use - basis.

\section{Advantages:}

(i) The details of cloud architecture could be different in different contexts.

(iii) Security is maintained by using account key (to authenticate and encrypt the private content), friend key (to secure channel between two friends), and content key (to protect an access control).

\section{Disadvantages:}

Availability: Various network failures and traffic congestion in the network may hinder the mobile users to connect to the cloud and access required services.

\section{Solutions to lack of availability issue:}

Using a discovery mechanism to find the nodes in the vicinity of a user whose link to cloud is unavailable [10]. The mechanism allows the user to connect to the cloud through the neighboring nodes in an ad hoc manner.

\section{Downsides of the solution proposed:} nodes.

The mechanism does not consider the mobility, capability of devices, and privacy of neighbouring

Alexey Rudenko et al. stated [4] that the cost of running a task locally is much higher than sending

those tasks to a server or cloud and receiving the outcome back. Battery life of a mobile is prolonged by remote 
process execution. Remote process execution facilitates moving processes from mobile to a server for better power management.

The server after executing the task sends the outcome back to the mobile for further processing. In the meantime, mobile executes other processes instead of just being idle and wasting the clock cycles of the processor. If the processes or tasks running on mobile consume lot of power and energy and the cost for moving processes to and fro server are low, then remote process execution is best alternative to save enrgy and prolong the battery life of the mobile.

\section{Advantages:}

Tasks can consume half the power which they are using initially if they are remotely executed.

\section{Disadvantages:}

The local card consumes huge amount of power if it is in receiving mode and thus increasing the cost due to high consumption of power.

\section{Solutions to the issue:}

To let the local card run in sleep mode and sync all the communications is a good option to conserve energy and reduce power cost. Additionally different portable computers with wireless cards can remain in idle state at a very low power cost. This also reduces power cost for remote process execution to a great extent.

\section{Downsides of proposed solution:}

To let the card into the sleep mode and return to active mode automatically and at the required time only is an obstacle which has to be handled using high precision algorithms.

Eduardo Cuervo et al. (2010) proposed a system called MAUI which enables offloading of mobile processes to a remote server [5]. MAUI is programmer friendly as it offloads the tasks to a remote server with least burden on the programmer. Which processes must run remotely is decided dynamically at the runtime.

\section{Advantages:}

(i) It uses code portability. Two versions are created of the mobile application in use. So that one runs locally on mobile and the other remotely on server to ignore the differences in the instruction set architecture between today's mobile devices and servers.

(ii) It uses programming reflection alongwith type safety. This helps in identification of the methods which need to be executed remotely and extraction of programming states of these methods.

(iii) Network transmission cost of a task can be determined by using serialization.

\section{Disadvantages:}

Power Save Mode while transferring a method's state remotely consumes large amount of energy.

\section{Solutions to the issues:}

To let the smart phone into the sleep mode will consume less power as compared to the power save mode.

\section{Downsides of the solution proposed:}

When the device is in the sleep mode, it can't perform any other operation. In power Save Mode it can perform other operations instead being idle.

Zhiyuan Li, Cheng Wang and Rong Xu (2001) considered handheld devices which are connected to a server via a wireless LAN. Offloading these handheld devices' computation to a remote server can save energy. To minimise the energy consumption by the programs, a partition scheme is applied to partition a program into server and client tasks so that the energy consumed by the program is reduced to a great extent. The whole program runs on the device itself if the set of server tasks is empty. A set of client tasks can never be empty as some I/O operations continuously run on mobile to facilitate the programs' working. One can easily run these client tasks on the server after configuring the handheld device as graphics terminal.

\section{Advantages:}

Programs are divided into server and client tasks with the help of partition scheme.

\section{Disadvantages:}

(i) If the set of server tasks is empty, the entire program runs on the handheld device consuming more power. 
(ii) The solution proposed of partition scheme is only viable for LAN.

ChengWang and Zhiyuan Li (2003) [11] proposed computation offloading scheme on handheld devices. The scheme divides a program into client and server programs as shown in Figure 2 . The client program runs on the client side and the server program runs on the server side. There are many applications in a handheld device that are time consuming. They run slow because of low computation speed of the device. The only feasible option is to offload full application or a part of it to a more powerful machine by partitioning it into client and server programs.

Partitioning of a program has a cost. This cost includes computation cost, data communication cost, run-time cost. Polynomial time algorithm is used to find optimal program partition for the given program.

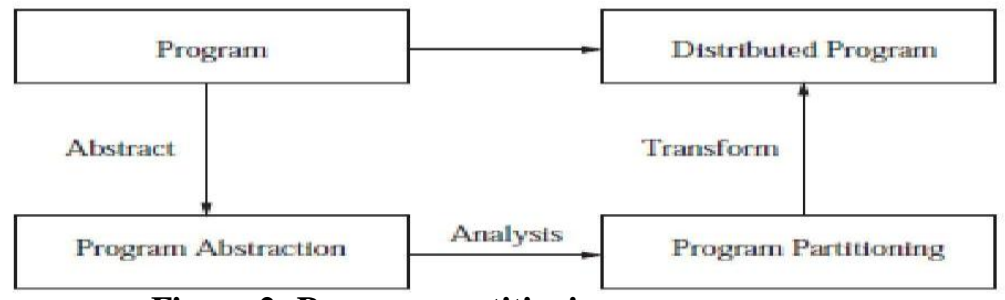

Figure 2: Program partitioning process

Advantages:

(i) Guaranteed correct distributed execution.

(ii) Optimal cost incurred.

\section{Disadvantages:}

Optimal program partitions vary with different program inputs. The optimal partition for one option may slow down the program execution for other inputs.

\section{Solutions to the issues:}

Computation offloading slow down may be improved by dynamic program partition according to the inputs and system information available at run time as shown in Figure 3.

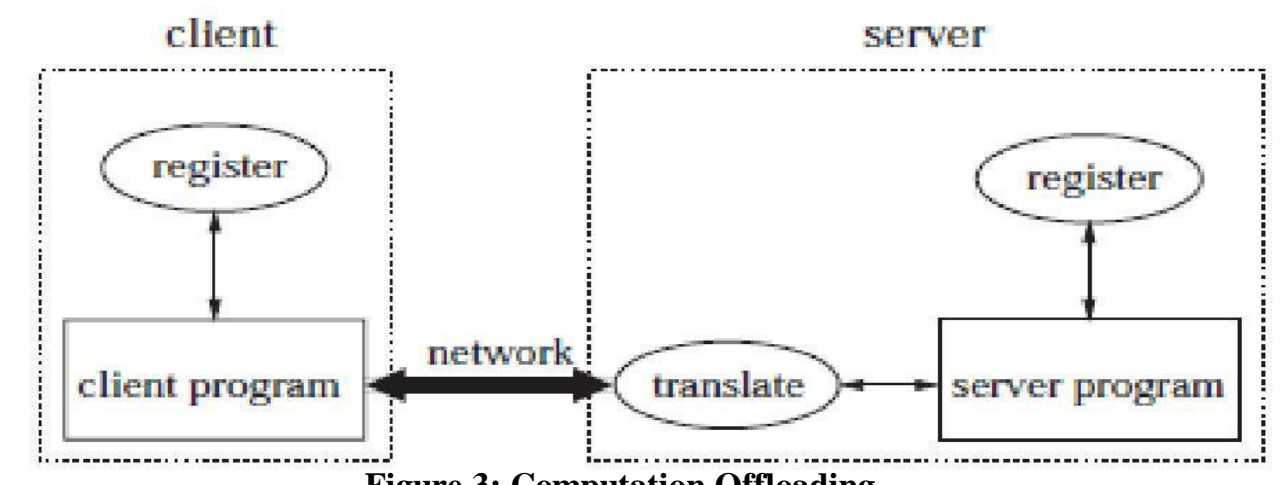

Figure 3: Computation Offloading

\section{Advantages Of Computation Offloading Into A Cloud}

3.1). Extended Battery Lifetime [2]: [6] evaluates large-scale numerical computations and shows that up to $45 \%$ of energy consumption can be reduced for large matrix calculation. In addition, many mobile applications take advantages from task migration and remote processing.

For example, offloading a compiler optimization for image processing [7] can reduce $41 \%$ of energy consumption of a mobile device. Also, using memory arithmetic unit and interface (MAUI) to migrate mobile game components [8] to servers in cloud can save $27 \%$ of energy consumption for the video game and $45 \%$ for the chess game.

\section{2). Improving data storage capacity and processing power [2]: Users can}

store/access large amount of data on clouds through mobile cloud computing. As all the data are processed on the cloud, there is no need for the mobile users to store data on the device. In this way a huge amount of storage space and energy of mobiles can be saved.

3.3). Improving reliability [2]: Data is stored on various computers to facilitate backups and archives. 
This reduces the possibility of loss of data on mobile devices as backups and archives are ready for data retrieval. integration.

3.4). Other advantages include High scalability, Dynamic provisioning, Multi -Tenancy, Ease of

\section{Overall Issues Related To Mobile Computing And Computation Offloading}

4.1). Low Bandwidth: One of the biggest issues of MCC is bandwidth. The lack of wireless networks with large speed and latency is a major issue in MCC.

4.2). Availability: Mobile users may not be able to connect to the cloud due to traffic congestion, lack of network coverage and various failures in the network.

4.3). Heterogeneity: WCDMA, WiMAX, GPRS, WLAN and CDMA2000 are various access technologies used by different mobiles to access services offered by the cloud.

4.4). Security: Security is one the greatest issues in MCC as all the data of different users can be stored in the same cloud. Maintaining privacy of the users' data from various threats is a major

4.5). Integrity: The data must not be altered due to external intrusion. To prevent integrity, security and privacy, authentication must be provided as service before a mobule user can offload his processes from the mobile.

4.6). Need for high speed Internet service: For MCC: to work efficiently and for computation offloading it is extremely necessary that the internet speed which the user is using with its mobile must be high. The only two options are $3 \mathrm{G}$ and $\mathrm{Wi}-\mathrm{Fi}$. 3G has advantages as its availability throughout and its speed. But it has disadvantage as in use of energy. $3 \mathrm{G}$ consumes huge amount of energy for accessing $3 \mathrm{G}$ network. This diminishes the goal to extend battery life. So the only viable option is Wi-Fi. It consumes less energy and has high bandwidth to enable computation offloading. But it has a disadvantage of lack of availability.

\section{Conclusion}

Some solutions are proposed here to overcome the issues related to mobile cloud computing. MCC provides mobile users with custom IT services in a simple, efficient and fast way to save energy. Security is a main issue in mobile cloud computing.

MCC is sustainable, but due to security issues less mobile users feel comfortable with mobile computing pertaining to theft, illegal modifications of private data. Security can be addressed with SSL Encryption to obstruct unwanted intrusion. Kerberos Network is a best method to maintain security in Computer Communication Networks like MCC. A Kerberos Network ensures both client-end and server -end security. It ensures that the user attempting to log in is a legitimate user and ensures the user that the application he is logging in, is a legitimate one. Graphical password is also an excellent method to prevent phishing viz. presenting oneself as a legitimate user by illegally acquiring username and password. Graphical password also reduces threats from bots which cause automatic internet crimes for which they are made. Graphical password is successor of captcha. In graphical password, user chooses a part of a graphic or an image as his password which only he can know. The graphic or the image is also of his choice. Hence, the inclusion of graphical

Password can greatly reduce security risks posed by MCC. To increase the use of MCC, a service provider must address the issue of compatibility. Compatibility issue occurs when existing IT infrastructure with the business is not compatible with the cloud technology. A Hybrid Cloud can be used to handle the compatibility issue. A hybrid cloud is the one in which a third party provider handles the restructure of the existing IT infrastructure. This will reduce the time and cost with personnel. In this paper many computation offloading methods have been analyzed. The mobile user can benefit from these partitioning schemes to enable computation offloading so that some of the computation intensive processes can be run on the cloud to minimize energy consumption and increase battery life of the mobile. Mobile cloud computing will become a viable option for enterprises in near future as it saves power by computation offloading. Businesses which will adopt this service earlier will definitely benefit in long run as it offers low total cost of ownership and high return of investment.

\section{References}

[1] "The NIST Definition of Cloud Computing". National Institute of Science and Technology 24 July 2011.

[2] Hoang T. Dinh, Chonho Lee, Dusit Niyato, and Ping Wang: A Survey of Mobile Cloud Computing: Architecture, Applications, and Approaches, pp. 1-25

[3] L. Liu, R. Moulic, and D. Shea. January 2011, Cloud Service Portal for Mobile Device Management, pp. 474.

[4] Alexey Rudenko, Peter Reiher, GerMd J. Popek and Geoffrey H. Kuenning: Saving Portable Computer Battery Power through Remote Process Execution, Vol. 2, pp. 19-20

[5] Eduardo Cuervo, Aruna Balasubramanian, Dae-ki Cho, Alec Wolman, Stefan Saroiu, Ranveer Chandra, Paramvir Bahl: June 2010, MAUI-Making Smartphones Last Longer with Code Offload, pp. 49-50.

[6] A. Rudenko, P. Reiher, G. J. Popek, and G. H. Kuenning: Saving portable computer battery power through remote process execution, vol. 2, no. 1, January 1998. 
[7] U. Kremer, J. Hicks, and J. Rehg. 2003. A compilation framework for power and energy management on mobile computers, pp. 115 $-131$

[8] E. Cuervo, A. Balasubramanian, et al.: June 2010. MAUI-Making Smartphones Last Longer with Code offload, pp. 49-62.

[9] C. Vecchiola, X. Chu, and R. Buyya: July 2009. Aneka-A Software Platform for .NET-Based Cloud Computing, pp. 267 - 295.

[10] G. Huerta-Canepa and D. Lee: 2010. A virtual cloud computing provider for mobile devices, no. 6 